\title{
Guidelines reduce the risk of brain-scan shock
}

\section{Responsibility for research is separate from a centre's duty of care to MRI volunteers.}

Sir - Your News story "Brain-scan ethics come under the spotlight ${ }^{n}$ (Nature 433 , $185 ; 2005)$ raises an important issue, which the Wolfson Brain Imaging Centre (WBIC) has addressed over the past decade as part of a clinical research programme that includes normal volunteers.

This policy was designed to avoid the distressing scenario highlighted by a previous correspondent ("How volunteering for an MRI scan changed my life ${ }^{p}$ Nature 434,$17 ; 2005$ ) and to be consistent with UK guidelines for the safe operation of magnetic resonance imaging (MRI) scanners.

In brief, we separate the responsibility for the research itself, which properly lies with the investigator, from the duty of care owed by the WBIC. In addition, various forms of insurance are in place depending on the nature of the study, supplemented by a policy for non-negligent harm.

All volunteers are offered counselling, which includes discussion of what will happen should an abnormality be detected on an MRI study. Our information/consent forms include the following paragraph:

"There is a chance of less than 1:100 that your MR scan may show a significant abnormality of which you are unaware. In such circumstances ... you will be referred to the appropriate specialist in consultation with your general practitioner, if that is what you would like. Such early detection has the benefit of starting treatment early but, in a small number of cases, may have implications for future employment and insurance."

A record is kept of MR exposure for each individual. All structural MRI studies are reviewed by a consultant neuroradiologist and a confidential report is generated, which is not included within the normal hospital information system.

Any significant abnormality is then discussed with the dinical director of the WBIC, who is a consultant neurosurgeon in active clinical practice.

If the abnormality may affect the validity of the research, the principal investigator is informed that it is no longer appropriate to include that individual in their study. The researcher is not told why and plays no further part in the process.

The volunteer is informed that there may be an abnormality and a full clinical MRI investigation is arranged if necessary. Volunteers are reassured that no communication will be made with their family doctor unless they so wish. This is to avoid details entering the medical records that might be used at some future date by life insurers and so on.

Finally, care has to be taken to ascertain that subjects are genuinely healthy volunteers. A very small number of volunteers are serial attenders at imaging centres worldwide. These people appear to use the system as a way of checking on their pathology, which they do not always declare at the time of screening. John D. Pickard, Jonathan H. Gillard Wolfson Brain Imaging Centre, University of Cambridge, Box 65 , Addenbrooke's Hospital, Cambridge CB2 2QQ UK

\section{DNA barcoding a useful tool for taxonomists}

Sir - The Consortium for the Barcode of Life (CBOL; see www.barcoding.si.edu) is an international initiative of natural history museums, herbaria, other biodiversity research organizations, governmental organizations and private companies which wish to promote the development and use of DNA barcoding.

CBOL is in complete agreement with the major point raised by M.C. Ebach and C. Holdrege in Correspondence, that ¿DNA barcoding is no substitute for taxonomy" (Nature 434, 697; 2005).

CBOL views barcoding as a useful tool for taxonomists and a cost-effective system with which non-specialists, such as border inspectors, can assign unidentified specimens to known species. In both cases, CBOL views barcoding as part of taxonomy and rejects the idea that DNA taxonomy will replace the practice of taxonomy based on diverse character sets.

Taxonomists have begun using DNA barcodes in three ways. First, barcoding can be used as a 'triage' tool for sorting new collections into units based on barcode sequences, of which some will belong to known species and others will be new to science. In CBOL's view, only expert taxonomists can resolve the relationship between new barcode-based clusters and species.

Second, DNA barcodes can also help assign specimens to known species in those cases where morphologic features are missing (in the case of immature, partial or damaged specimens) or misleading (as in sexually dimorphic species). Third, barcodes can also be used as a supplement to other taxonomic datasets in the process of delimiting species boundaries.

Ebach and Holdrege are correct in stating ${ }^{\alpha} \mathrm{DNA}$ barcoding generates information, not knowledge". CBOL believes that this information can make systematists and the consumers of taxonomic information more knowledgeable. Therein lies its potential value.

David E. Schindel, Scott E Miller

Consortium for the Barcode of Life, National Museum of Natumal History, Smithsonian Institution, PO Box 37012, MRC-105, Washington, DC 20013-7012, USA

\section{Debate to be had on best practice in mentoring}

Sir - I much appreciated your Editorial "Joys of (top-notch) supervision" (Nature 434, 421; 2005). Having supervised 35 successful $\mathrm{PhD}$ students - and resisting the temptation to write a book on the subject - I believe that there is a debate to be had on good practice in student project mentoring and the problems of identifying and avoiding poor supervision.

For example, universities have procedures for recognizing academic staff who are qualified to supervise research students, but, in my experience, they lack adequate processes for dealing with poor supervisors.

To focus on one particular point, that of co-authorship of publications with the supervisor: the supervisor should indeed have contributed to the scholarship substantially beyond the baseline of normal supervisory support.

However, practice varies between disciplines, and in certain areas of the physical and biomedical sciences it is the norm for the supervisor's name to appear on any publication arising from a student's research.

Alan Lord

University College London,

Gower Street,

London WC1E 6BT, UK

\section{correspondence}

Contributions to Correspondence may be submitted to corres@nature.com. They should be no more than 500 words in length, and ideally shorter. Published contrbutions are edited. 